\title{
Simulation and analysis of force on connection of casing thread under alternating load of gas storage
}

\author{
Hanhua Song ${ }^{1}$, Wei Tian ${ }^{1}$, Chen $\mathrm{Li}^{1}$, Zaishun $\mathrm{Li}^{1}$, Lianlian Fan ${ }^{1}$, Jian $\mathrm{Lu}^{2}$ and Yufeng Gong ${ }^{3, *}$ \\ ${ }^{1}$ Oil and Gas Technology Research Institute of Changqing Oilfield Company \\ ${ }^{2}$ Changqing Oilfield Company gas Field Development Division \\ ${ }^{3}$ School of Energy, Chengdu University of Technology, Chengdu, China
}

\begin{abstract}
Underground gas storage as the main system of natural gas peak regulation, its construction demand continues to grow in the world. Different from other common oil and gas well strings, the service time of the gas storage string is longer and the service environment is worse, which poses a greater challenge to the integrity design of the string joint. In this paper, starting from the most important connection and sealing performance of the pipe string joint, aiming at the special threaded joint suitable for the gas storage pipe string, based on the analysis of the force and deformation of the gas storage pipe string, the joint connection strength and sealing performance of the pipe string joint under the axial load, internal and external pressure and longterm alternating load are considered. Based on the FINITE element model of VAM TOP special threaded joint, the deformation and sealing behavior of the joint under different composite loads were numerically simulated, and the variation law of joint connection stress and sealing contact pressure with load was obtained, and the most vulnerable failure position and the most dangerous working condition were analyzed.
\end{abstract}

\section{Introduction}

With the continuous growth of the demand for natural gas, the phenomenon of insufficient natural gas supply often occurs. In order to adjust the balance of natural gas supply and demand, it is necessary to use natural gas reserve system. At present, underground gas storage has become the most important way of natural gas storage and peak regulation in the world because of its advantages of high safety factor and large storage capacity [1-5]. Different from the common oil and gas well pipe string, the underground gas storage pipe string has been in service for more than 30 years, and the service environment is very bad. The alternating load caused by the long-term gas injection and production process poses a severe challenge to the design of pipe string, especially the design of pipe string joint. As the most critical part of the whole string, the joint of the string is also the weakest link, which can easily cause gas leakage due to failure [6-8].

There are two main reasons for the failure of underground gas storage pipe string joint: connection failure and sealing failure. Connection failure mainly includes the following forms :(1) trip failure (2) fracture failure (3) shear failure (4) over torsion failure. Seal failure refers to the gas leakage phenomenon of threaded joint due to various reasons such as machining accuracy, operation mode and working condition. In view of the above failures, it is necessary to study the connection and sealing integrity of threaded joint in order to maintain the operation safety of gas well in gas storage. The research methods of mechanical properties of threaded joints mainly include analytic method, full-size test method and finite element method.

\section{Theoretical Basis}

(1) Axial force calculation

In the early stage of production, the injection-production string is set inside the production tubing by a packer, and changes in the external extrusion pressure and internal pressure can be translated into axial force by the bulging effect or the piston effect. In the production process, the internal pressure in the string fluctuates and alternates with the frequent alternation of injection-production cycle, resulting in the change of external extrusion pressure and axial force. During gas injection and recovery, the formation volume expands or compacts the string in an axial direction. At the same time, the inner diameter of the pipe string varies, and the temperature, density, and flow rate of the pipe string fluctuate, which will impose additional axial load on the pipe string.

Therefore, the axial force of the gas storage pipe string is the sum of the superimposed axial force caused by dead weight, temperature effect, expanding diameter effect and ground stress during gas injection and production.

The axial tension generated by the dead weight of the tube body can be calculated by:

$$
G=\rho_{1} g(L-z)
$$

\footnotetext{
* Corresponding author: gongyufeng1995@stu.cdut.edu.cn
} 
Where, $\rho 1$ is the oil pipeline density, $\mathrm{kg} / \mathrm{m}, \mathrm{L}$ is the tubing length, $\mathrm{m}, \mathrm{z}$ is the calculation point depth, $\mathrm{m}$.

(2) Calculation of internal pressure strength

The internal compression strength of pipe string under combined load can be obtained by standard SY/T53222000:

$$
p_{c_{a}}=p_{b o}\left[\frac{r_{i}^{2}}{\sqrt{3 r_{o}^{4}+r_{i}^{4}}}\left(\frac{\sigma_{a}+p_{0}}{Y_{p}}\right)+\sqrt{1-\frac{3 r_{o}^{4}}{3 r_{o}^{4}+r_{i}^{4}}\left(\frac{\sigma_{a}+p_{0}}{Y_{p}}\right)^{2}}\right]
$$

(3) Calculation of tensile strength

The gas storage pipe string still complies with the fourth strength criterion under the combined action of internal pressure, non-uniform external load and axial force, so the tensile strength of the pipe string under combined load is:

$$
T_{a}=10^{-3} \pi\left(P_{i} r_{i}^{2}-P_{0} r_{0}^{2}\right)+\sqrt{T_{0}^{2}+3 \times 10^{-6} \pi^{2}\left(P_{i}-P_{0}\right)^{2} r_{0}^{4}}
$$

$\mathrm{Ta}$-- Triaxial tensile strength of pipe body, KN;

$\mathrm{Pi}$-- Equivalent external pressure of tubing, $\mathrm{MPa}$,

$\mathrm{Po}$-- Tubing internal pressure, $\mathrm{MPa}$,

$\mathrm{Ri}$-- Tubing radius, $\mathrm{cm}$;

Ro -- Outer tubing radius, $\mathrm{cm}$.

(4) Calculation of compressive strength

Equivalent yield strength under axial force can be calculated by the following formula:

$$
Y_{p a}=\left[\sqrt{1-0.75\left(\sigma_{a} / Y_{p}\right)^{2}}-0.5\left(\sigma_{a} / Y_{p}\right)\right] Y_{c_{a}}
$$

Ypa -- Collapse pressure under axial tensile stress, MPa; Yca -- Uniaxial collapse pressure, MPa;

$\sigma$ a -- Axial stress, MPa;

Yp -- Pipe body yield strength, MPa.

Because of the working condition of gas storage, it is easy to collapse under combined load. Then the compressive strength of tubing under combined load is:

$$
P_{c a}=P_{c o}\left\{\left[1-\frac{3}{4}\left(\frac{\sigma_{a}+P_{i}}{Y_{p}}\right)^{0.5}-\frac{\sigma_{a}+P_{i}}{2 Y_{p}}\right]\right\}
$$

During gas recovery, axial pressure will be exerted on the tubing due to the decline of the inner layer of the well. At this time, the tubing compressive strength is:

$$
P_{c a}=P_{c o}\left\{\left[1-\frac{3}{4}\left(\frac{\sigma_{a}+P_{i}}{Y_{p}}\right)^{0.5}+\frac{\sigma_{a}+P_{i}}{2 Y_{p}}\right]\right\}
$$

\section{Modeling process}

The working environment of tubing is bad and the alternating load is large. Because the joint part itself is relatively weak, coupled with its sectional shape change, there is a high alternating stress, stress concentration is serious, is the most dangerous part of the force. The model established in this paper is shown in Figure 1 and Figure
2. V-038r, taper $1: 4$, elastic modulus $\mathrm{E}=200 \mathrm{GPa}$, Poisson's ratio $=0.3$, density $7850 \mathrm{~kg} / \mathrm{m} 3$.
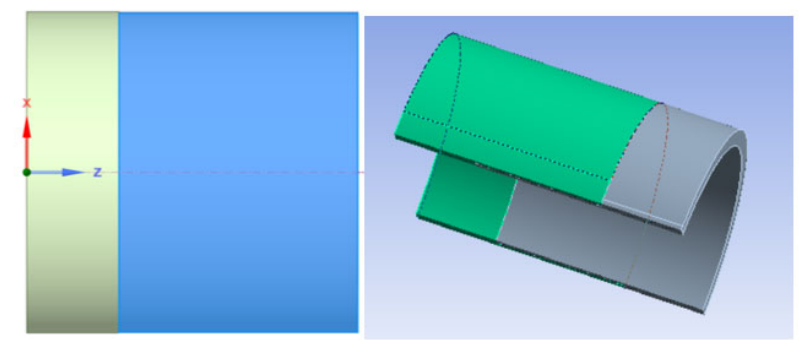

Figure 1. Model of thread connection

Set contact surface: Friction contact is used for the two geometers. There are 239320 nodes and 132,494 cells in the volume and contact grids.

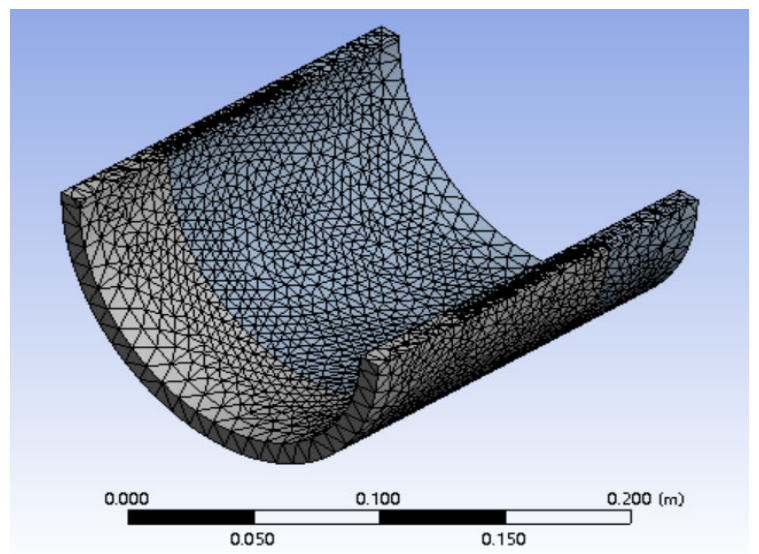

Figure 2. Meshing result diagram

Load added: The external extrusion force of the pipe string is $25 \mathrm{MPa}$, the internal pressure is respectively set as $20 / 15 / 10 \mathrm{mpa}$ (pressure in different injection and production processes), and the axial tension is set as $10 / 20 / 30 / 40 / 50 \mathrm{mpa}$ (simulated axial loads are $99 \mathrm{KN}$, $198 \mathrm{KN}, 297 \mathrm{KN}, 396 \mathrm{KN}$ and $495 \mathrm{KN}$ ).

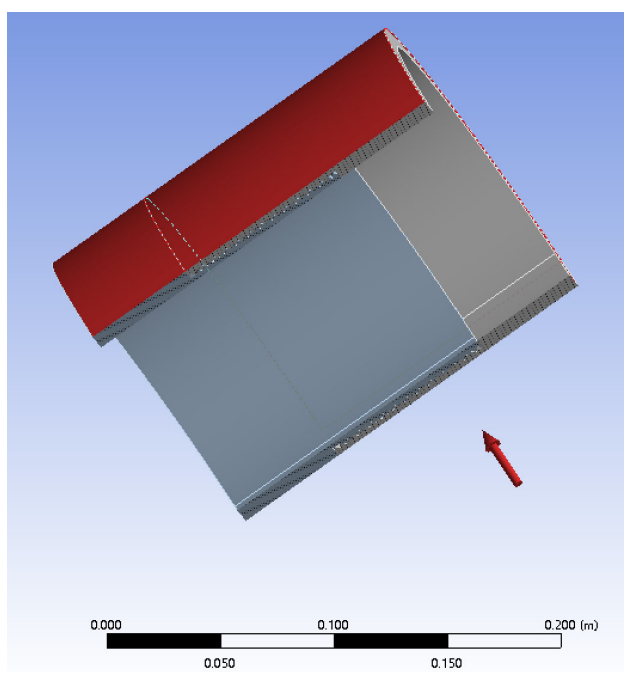

Figure 3. Schematic diagram of load loading 


\section{Discussion}

Stress concentration occurs at the last thread of female thread, the maximum deformation is $3.0 \mathrm{~mm}$, the maximum equivalent stress is $584.8 \mathrm{mpa}$, the contact compressive stress is very large, there is a risk of fatigue damage.

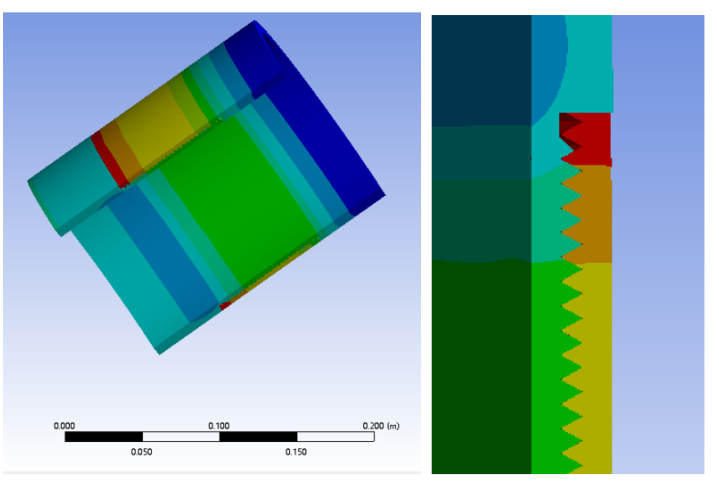

Figure 4. Stress analysis of thread connection

The stress concentration occurs at the last thread of female thread, the maximum deformation is $2.9 \mathrm{~mm}$, the maximum equivalent stress is $565.8 \mathrm{MPa}$, the maximum deformation increases, but the maximum equivalent stress decreases.

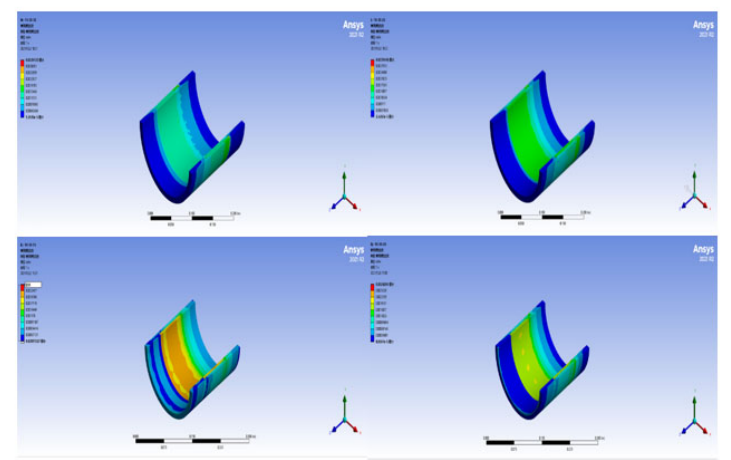

Figure 5. Strength analysis under different internal pressures (external pressure $=30 \mathrm{MPa}$, axial pressure $=30 \mathrm{MPa}$ )

Under different internal compressive strength, the maximum strain is $3.41 \mathrm{~mm}$, the minimum strain is $2.51 \mathrm{~mm}$, the maximum stress is $668.62 \mathrm{MPa}$, and the minimum stress is $488.23 \mathrm{MPa}$.

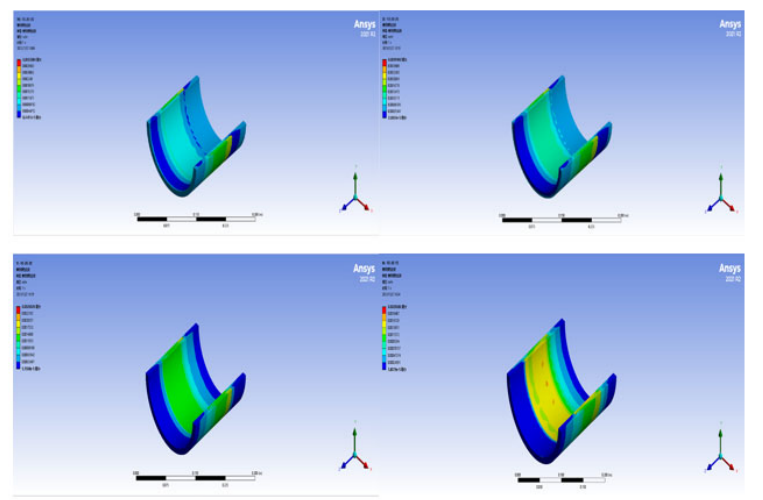

Figure 6. Strength analysis under different internal pressures (external pressure $=30 \mathrm{MPa}$, axial pressure $=30 \mathrm{MPa}$ )
The maximum equivalent stress of $666.8 \mathrm{mpa}$ exceeds the thread connection limit stress, and the thread fatigue failure occurs.Two-dimensional binomial fitting was carried out, the fitting error R2 $=0.9947$, and the fitting effect was relatively good.

$$
\begin{gathered}
\mathrm{P}(\text { Pout }, \text { Pin })=-403.2+44.89 * \text { Pout }+18.86 * \text { Pin } \\
-0.3409 \text { Pout } 2-0.5999 \text { Pout * Pin }
\end{gathered}
$$

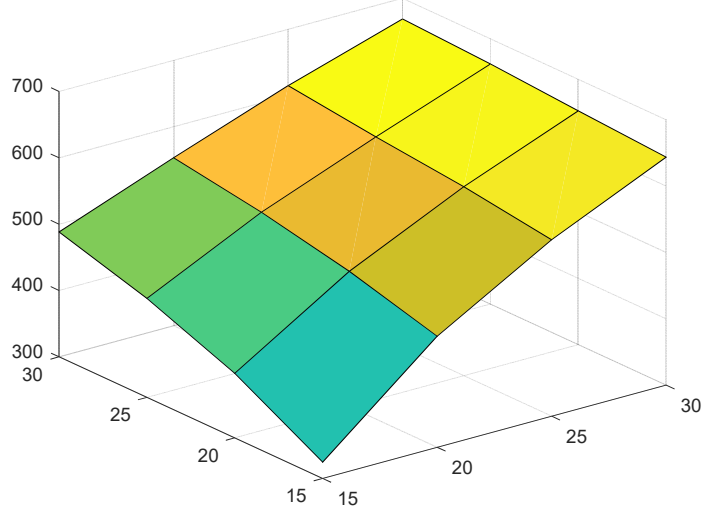

Figure 7. Schematic diagram of fitting $3 \mathrm{D}$ results

\section{Conclusion}

(1) Under the same external pressure, with the increase of internal pressure, the maximum strain increases gradually, the maximum stress increases gradually, and the limit life decreases.

(2) The thread connection needs to be considered, the lower part needs to check the compressive strength and extrusion strength. The upper part needs to be checked for axial tension.

\section{References}

1. Reitenbach, Viktor, et al. "Influence of added hydrogen on underground gas storage: a review of key issues."Environmental Earth Sciences. 73.11 (2015): 6927-6937.

2. Mazarei, Mehdi, et al. "The feasibility analysis of underground gas storage during an integration of improved condensate recovery processes."Journal of Petroleum Exploration and Production Technology. 9.1 (2019): 397-408.

3. ZHENG, Dewen, et al. "Key evaluation techniques in the process of gas reservoir being converted into underground gas storage."Petroleum Exploration and Development. 44.5 (2017): 840-849.

4. Davarpanah, Afshin, Mehdi Mazarei, and Behnam Mirshekari. "A simulation study to enhance the gas production rate by nitrogen replacement in the underground gas storage performance."Energy Reports. 5 (2019): 431-435.

5. Wang, Tongtao, et al. "Determination of the maximum allowable gas pressure for an underground gas storage salt cavern-A case study of Jintan, 
China." Journal of Rock Mechanics and Geotechnical Engineering. 11.2 (2019): 251-262.

6. Liu, Jun, et al. "Nonlinear flow-induced vibration response characteristics of leaching tubing in salt cavern underground gas storage." Journal of Energy Storage. 41 (2021): 102909.

7. Wang, Tongtao, et al. "Safe distance between debrining tubing inlet and sediment in a gas storage salt cavern."Journal of Petroleum Science and Engineering. 196 (2021): 107707.

8. Bai, Mingxing, et al. "Well completion issues for underground gas storage in oil and gas reservoirs in China."Journal of Petroleum Science and Engineering. 171 (2018): 584-591. 